\title{
Normal relation between heart rate and cardiac repolarisation in sudden infant death syndrome
}

Vernon T Wynn, David P Southall

\begin{abstract}
Objective-To determine whether there was a difference in the relation between heart rate and $R T$ intervals in infants who later died of sudden infant death syndrome (SIDS) and controls.

Design-A blinded, computer analysis of prospectively acquired physiological data on SIDS cases and controls.
\end{abstract}

Setting-Physiological data obtained from infants at home (collaborative analysis National Heart and Lung Institute and Exeter University).

Participants-Nine fullterm infants who subsequently died of SIDS and 10 surviving controls matched for age and birth weight.

Interventions-24 hour tape recordings of the electrocardiogram at home between three and 11 weeks of age.

Main outcome measures-Mean value of the constant $b$, which relates by linear regression the $\log$ of each $R T$ interval to the $\log$ of its preceding $R R$ interval, and any difference between SIDS cases and controls.

Results-The mean (2SEM) values for $b$ were $0.20(0.03)$ for the 10 controls and $0.19(0.03)$ for the nine SIDS infants. Cyclical correlations between $R T$ and $R R$ intervals of varying strength were identified in both SIDS infants and controls.

Conclusions-Infants who subsequently died of SIDS did not show an impaired ability to modify $R T$ intervals in response to change in heart rate.

In our previous prospective studies of electrocardiograms from infants who died of sudden infant death syndrome (SIDS) we did not find any predictive lengthening of the QT interval. ${ }^{12}$ The purpose of this present analysis was to evaluate the response of the QT interval to variations in heart rate. We hypothesised that a defect in this response might indicate a vulnerability to dangerous ventricular arrhythmias. A similar analysis has been reported by Sadeh et $a l^{3}$ of 24 hour electrocardiographic recordings collected by us $^{1}$ from 10 infants who subsequently died of SIDS. The ability to shorten their QT interval in response to an increasing heart rate was reported to be impaired in five of their 10 cases. As a result of their findings Sadeh et al argued that "sudden death in these cases may have resulted from defective modulation of cardiac repolarisation". ${ }^{3}$ In contrast our own analysis of the same data has produced different results. In the light of potentially harmful preventive therapies, we consider it important to describe our own analysis. We then discuss the possible reasons for this evident conflict between our findings and those of Sadeh et al. ${ }^{3}$

\section{Patients and methods}

We analysed segments from ten 24 -hour cassette tape recordings of electrocardiograms obtained prospectively on nine full term victims of SIDS aged 16-63 days at the time of recording (birth weights from 2.35 to $4.79 \mathrm{~kg}$ ) (table 1). Ten similar recordings on 10 surviving fullterm controls matched for postnatal age (21-75 days) and birth weight (2.64 $4.00 \mathrm{~kg}$ ) were similarly studied (table 1 ). We examined the same recordings from SIDS cases as Sadeh et al. ${ }^{3}$ However, Sadeh et al included only data from midnight to $6 \mathrm{am}$; we intentionally used complete data from the entire 24 hour period to include daytime and night time activity.

The waveforms in the prospective study yielding these data ${ }^{1}$ were obtained from two electrodes on the chest wall. The frequency content of the resulting signals was $0.5-70 \mathrm{~Hz}$ after processing through the tape replay system (Oxford Medilog PB2). Details of the technique have been previously reported in detail. ${ }^{45}$ In brief, electrocardiograms from the Medilog recorder together with the simultaneously recorded $60 \mathrm{~Hz}$ calibration signals were

Table Details of recordings and infants

\begin{tabular}{lll}
\hline Age (days) & Birth weight $(g)$ & Gestation $(w k)$ \\
\hline \multicolumn{3}{c}{ SIDS cases } \\
16 & 2780 & 39 \\
$24^{\star}$ & 3770 & 40 \\
$63^{\star}$ & 3770 & 40 \\
30 & 2350 & 40 \\
40 & 3030 & 40 \\
40 & 3960 & 41 \\
41 & 4790 & 40 \\
47 & 2920 & 40 \\
52 & 2810 & 39 \\
61 & 2980 & 40 \\
& & \\
& Controls & \\
21 & 3660 & 42 \\
36 & 3700 & 42 \\
75 & 2640 & 38 \\
37 & 3740 & 38 \\
39 & 3690 & 40 \\
42 & 3300 & 40 \\
42 & 3230 & 39 \\
42 & 3950 & 40 \\
55 & 3800 & 38 \\
61 & 4000 &
\end{tabular}

$\star$ Same infant.
Dr David P Southall National Heart and Lung Institute, Royal Brompton Hospital, Sydney Street, London SW3 6NP.

Accepted for publication 10 September 1991
Department of Exeter, Exet

The National Heart Royal Brompton

Hospital, London

D P Southall 


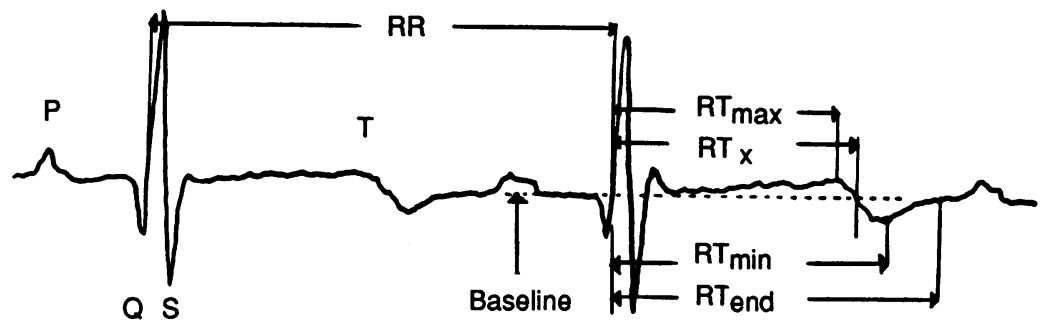

$100 \mathrm{~ms}$

Figure 1 An example of two electrocardiographic complexes from one of the controls. $R T_{\max }, R T_{x}, R T_{\min }$, and $R T_{\text {md }}$ behave in a similar fashion to the $Q T$ interval with changes in the immediately preceding $R R$ interval. $R T_{x}$ was chosen to represent ventricular repolarisation time. proportional to $\mathrm{RT}_{\mathbf{x}}$. (Linear regression analysis was used to fit equations to plots of $R_{\text {end }}$ against $R R$ and plots of $R T_{x}$ against $R R$ from data obtained in the previously reported study. ${ }^{45}$ The mean (SEM) slopes and intercepts respectively were $0.35(0.03)$ and 57 (12) ms for $\mathrm{RT}_{\mathrm{x}}$ against RR and 0.37 (0.03) and 70 (12) $m$ s for $\mathrm{RT}_{\text {end }}$ against RR).

If it is assumed that $R T_{x}$ and QT are directly proportional the following equation can be written:

$$
\log _{\mathrm{e}}\left(R \mathrm{~T}_{\mathrm{x}}\right)=\mathrm{b} \log _{\mathrm{e}}(\mathrm{RR})+\mathrm{k} \text { equation } 1
$$

where $k$ is the intercept and where $b$ is a constant that relates the $\mathrm{RT}_{\mathrm{x}}$ to the $\mathrm{RR}$ according to a power law.

From each 24 hour tape recording of SIDS cases and controls, a technician who did not know whether the data originated from a SIDS case or a control selected 8-10 segments using the data collected in the earlier study. ${ }^{45}$ Each segment consisted of 63 consecutive cardiac cycles with a high signal to noise ratio. The number of segments was chosen to ensure statistical significance for each measurement of $b$ and $k$. The number of cardiac cycles per segment (63) was chosen to match the program within the eight-bit microprocessor and was consistently large enough to include several short-term cycles in heart rate variability. The segments from each infant, whether a control or SIDS case, were chosen so as to provide as wide and evenly spaced a range of $R R$ intervals as possible. The computer data were in the form of approximately 1000 numerical tables, each table consisting of 63 pairs of consecutive $R R$ and $R T_{x}$ values. Only those tables that showed no trace of artefact (low noise) when observed on an oscilloscope as they were being loaded into the computer were selected by the independent technician.

The RR interval range for each of the segments of 63 heart beats in the controls varied from 14 to $288 \mathrm{~ms}$; that is, in one segment the RR interval varied by only $14 \mathrm{~ms}$ over the 63 beats while at the other extreme the $R R$ interval varied by 288 ms over the 63 beats. The overall range for the SIDS cases varied from 8 to $294 \mathrm{~ms}$.

Correlation coefficients and values of $b$ were obtained by fitting equation 1 to the $\mathrm{RT}_{\mathrm{x}}$ values and their corresponding $R R$ values by linear regression analysis for both SIDS cases and controls (fig 2A, B, and C). These values of $b$ were also plotted against the individual ranges of RR intervals within each 63 beat segment for all recordings in both controls and SIDS cases (fig 3). Time series plots of successive RR and $\mathrm{RT}_{\mathrm{x}}$ intervals were then obtained for each 63 beat segment analysed. much reduced compared with those of the remaining three measurements. The $\mathrm{RT}_{\mathrm{x}}$ interval also had the advantage of being less affected by those electrocardiographic waves that were so short that the $P$ wave was mixed in with the end of the $T$ wave.

The small size of the $Q R$ interval and the close relation between changes in the preceding RR intervals and both $\mathrm{RT}_{\mathrm{x}}$ and $\mathrm{RT}_{\text {end }}$ indicated that a good estimate of the relation between QT and RR intervals could be obtained by assuming that QT interval duration is directly

\section{Results}

The mean (2 SEM) value of $b$ for the SIDS cases was $0.20(0.02)$ when all 10 SIDS recordings were used and $0.19(0.03)$ when analysis was limited to the later of two recordings on one the mean value of $b$ was $0.20(0.03)$. The time series plots of $R R$ and $R T_{x}$ intervals behaved in of the SIDS cases. For the surviving controls 

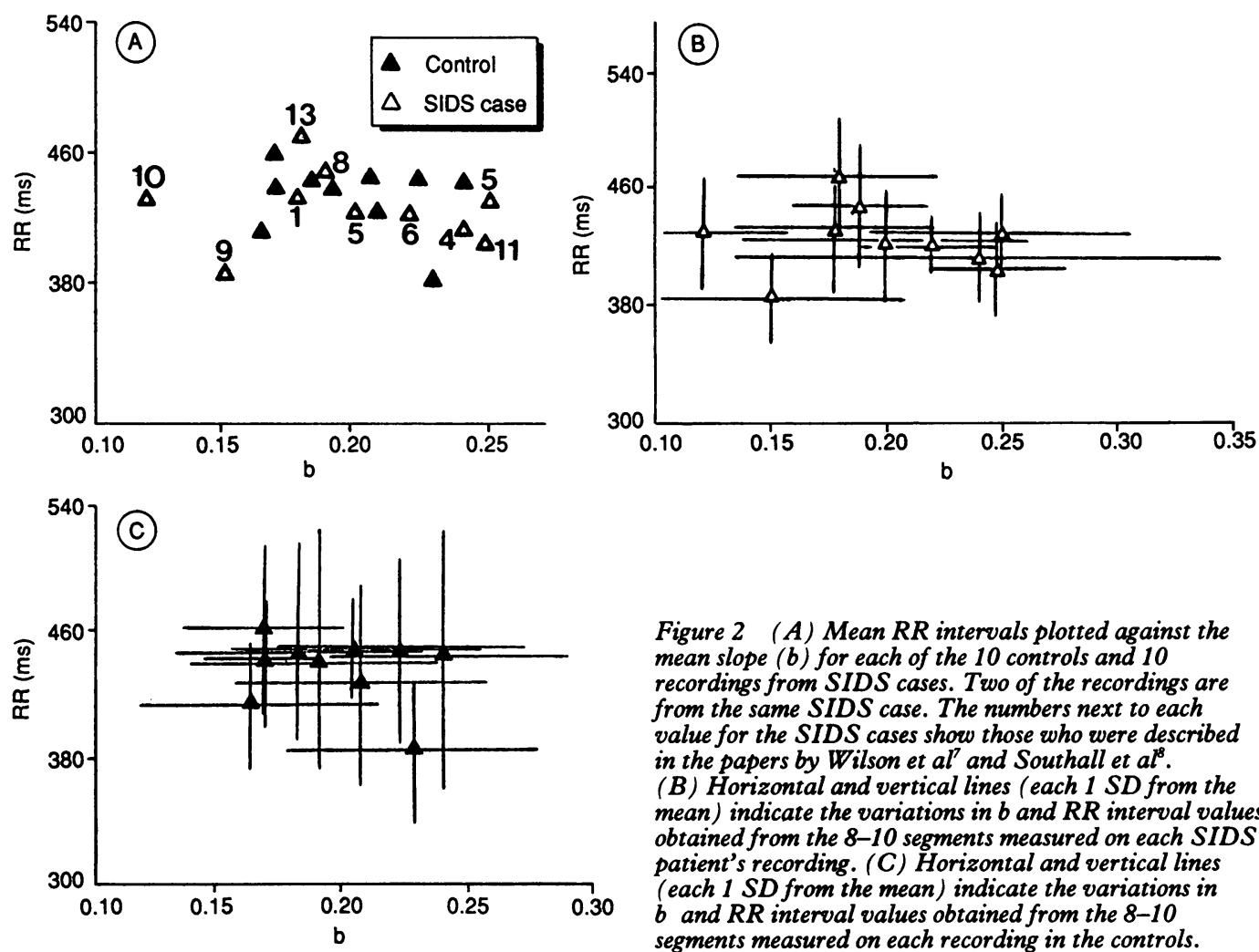

Figure 2 (A) Mean RR intervals plotted against the mean slope (b) for each of the 10 controls and 10 recordings from SIDS cases. Two of the recordings are from the same SIDS case. The numbers next to each value for the SIDS cases show those who were described in the papers by Wilson et al and Southall et al ${ }^{s}$. (B) Horizontal and vertical lines (each 1 SD from the mean) indicate the variations in $b$ and $R R$ interval values obtained from the 8-10 segments measured on each SIDS patient's recording. (C) Horizontal and vertical lines (each $1 S D$ from the mean) indicate the variations in $b$ and $R R$ interval values obtained from the 8-10 segments measured on each recording in the controls.

such a similar fashion for both controls and SIDS cases that they could not be distinguished by statistical analysis. Periodicities of varying amplitude and correlation were frequently seen in the different segments from each infant, irrespective of whether they were victims or controls. Figure $2 \mathrm{~A}$ shows the data for the nine cases of SIDS (10 recordings) and 10 controls, constructed by plotting the mean $R R$ interval for all segments on each infant against the mean value of $b$ for the same segments. The numbers set against the SIDS patients indicate those infants in whom heart rate ${ }^{7}$ and heart rate variability $^{8}$ were measured in earlier studies. Figure 2A clearly shows that it is not possible to distinguish SIDS cases from their controls by the value of $b$. The range of the mean values of $b$ in both SIDS cases and controls is 0.11 to 0.25 .

Figure 2B shows the variability in terms of standard deviation from the mean for the values of both $b$ and $R R$ intervals for all recorded segments from infants dying suddenly. Values in the controls were virtually identical (fig $2 \mathrm{C}$ ).

Figure 3 shows the mean values of $b$ for both SIDS cases and controls plotted against the mean $R R$ interval ranges. The mean value of $b$ for the RR range of $212 \mathrm{~ms}$ is the mean of seven points. All the other mean values have been calculated by grouping the values of $b$ and their respective RR ranges into sets of at least 10 . There was no significant difference between the SIDS group and the controls in this graph. As the range of $R R$ intervals increased the value of $\mathrm{b}$ fell.

Cyclical behaviour in the relation between RT and RR intervals was as common in the controls as in the SIDS cases.

\section{Discussion}

Prospective identification of infants at risk of sudden death is a desirable objective on which to base preventive care. However, incorrect predictors may prompt harmful intervention, especially if cardioactive treatment is being considered. This is why the results presented by Sadeh et al, ${ }^{3}$ with their implications concerning prediction, must be confirmed or rejected. Unfortunately, only one prospective data base is available on which these results can be checked and that is the original 24 hour electrocardiograms obtained by our group. Sadeh et al's report was published before we

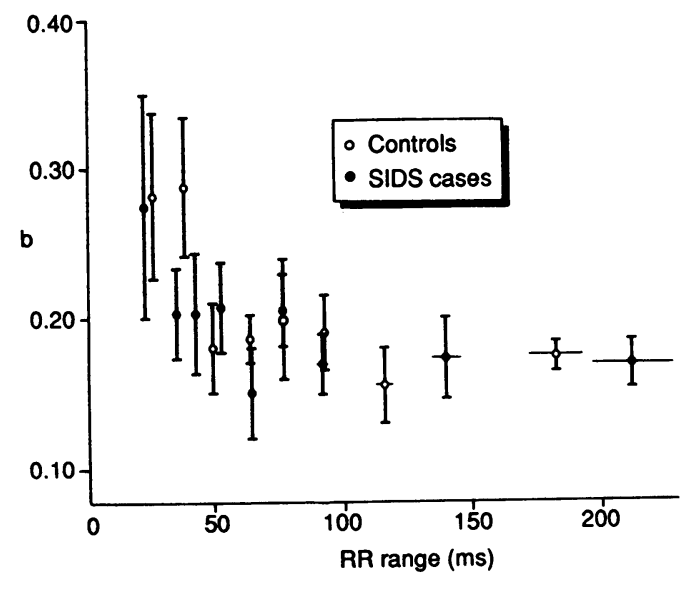

Figure 3 Plot of mean values of $b$ for both SIDS cases and controls against the mean $R R$ interval ranges. The value of $b$ at the $R R$ interval range of $212 \mathrm{~ms}$ is the mean of seven points. All of the other mean values were calculated by grouping the values of $b$ and their respective $R R$ ranges into sets of at least 10. The horizontal and vertical lines are SEMs. 
had completed an analysis of our data with exacting attention to several technical and mathematical artefacts that could easily invalidate conclusions about the association between ventricular electrical activity and the incidence of sudden infant death. This analysis is now complete and unlike Sadeh et al we found no consistent differences between SIDS cases and controls.

The analysis in this paper differs from that carried out previously by our group. ${ }^{45}$ Earlier work was based on mean values of the intervals in the electrocardiogram obtained by simply averaging over 63 consecutive heart beats. In the present study we undertook the more difficult investigation of the relation between individual $R T$ ventricular repolarisation times and each preceding $R R$ interval (fig 1 ).

This analysis of the time-series relation between $R T$ and $R R$ intervals did not show any difference between recordings from SIDS infants and their surviving controls. These results do not, therefore, support the hypothesis that a disordered relation, as measured from the surface electrocardiogram, between ventricular repolarisation time and heart rate influences subsequent sudden unexplained infant death. Moreover, within each infant's recording, whether they were SIDS cases or controls, some segments were found to show a strong cyclical correlation between $R T$ and $R R$ intervals while others showed no such relation. Sadeh et al inferred that unlike SIDS cases the controls always showed this strong correlation.

The spread of values for the constant $b$ (which relates by linear regression the $\log$ of each RT interval to the log of its preceding RR interval) in both controls and SIDS cases in this present study was less $(0 \cdot 11-0.25)$ and the mean value of $b$ for controls was greater $(0 \cdot 20)$ than values found by Sadeh et $a l^{3}$ (their range was $0.05-0.25$, and their mean for controls was 0.13). These present findings, however, do accord with those of Sadeh et $a l^{3}$ in suggesting that Bazett's formula ${ }^{9}$ (where $b=0.50$ ) provides an inappropriate method for correcting the QT interval for heart rate in this age group.

The following differences between SIDS cases and controls were reported by Sadeh $e t$ $a l .^{3}$ Firstly, according to the equation QT = $C(R R)^{b}$, their mean (1 SD) values for $b$ were $0.096(0.032)$ in the SIDS cases and 0.134 $(0.043)$ in controls $(p<0.02)$. Secondly, their illustrated time series of QT intervals plotted against the immediately preceding $R R$ intervals from 500 consecutive heart beats showed, in a single control recording, highly correlated cyclical variations in both intervals. There was a much smaller correlation in a similar plot from a single time series from a SIDS case. Presumably these two plots were included to lend weight to differences that they concluded existed between the controls and five of the SIDS subjects. Finally, in their plot of $b$ against mean $R R$ intervals for each segment of the recording analysed for the 10 SIDS cases and 29 controls, five of the 10 sudden death values for $b$ fell outside the $95 \%$ confidence interval for the controls.
There are several possible explanations for the differences between our results and those of Sadeh et al. ${ }^{3}$ Firstly, to "improve the reliability of their statistical comparisons" Sadeh et al ${ }^{3}$ selected their segments on the basis that the $R R$ intervals within each segment varied by more than $100 \mathrm{~ms}$. As shown in fig 3, this could result in lower values of $b$. If values of $b$ in this present report are limited to those segments where the $R R$ interval range is $>100 \mathrm{~ms}(>45$ sets of points), mean ( 2 SEM) values of $b$ are $0.16(0.03)$ for controls and $0.17(0.02)$ for SIDS cases. These values are reasonably close to the control measurements of Sadeh $e t a l^{3}$ $(0.13(0.04)(S D))$. The small remaining difference between the control values of $b$ is possibly a result of the two different methods by which each study chose to compensate for the difficulties in estimating the end of ventricular repolarisation. A second possible explanation for the different results is the high pass $(10 \mathrm{~Hz}$ cut-off) filter used by Sadeh et al $^{3}$ before the measurement of QT and RR intervals. This filter produces a phase difference between input and output signals which decreases as the frequency components of the input signal increase. Therefore, with higher heart rates containing correspondingly higher frequencies, there would be a smaller timing error between the true QT and their measured QT interval. This would result in the changes in duration of the QT interval appearing to be smaller in response to changes in $R R$ interval at the higher heart rates. For this reason we did not use a highpass filter but rather a high quality frequency modulated tape copy directly from the original 24 hour cassette recordings. Contrary to the statement made by Sadeh et $a l^{3}$ the frequency response of the signals after replay through the Oxford Medilog PB2 system is $0 \cdot 5-70 \mathrm{~Hz}$ not $10-100 \mathrm{~Hz}$. Nevertheless, as previously reported by us, ${ }^{1}$ the frequency response of the original cassette tape recording system is not ideal for low frequency signals such as the $T$ wave (diagnostic electrocardiogram recordings have bandwidths from 0.05 to $100 \mathrm{~Hz}$ ).

A third reason for the discrepancy between these present results and those of Sadeh et al ${ }^{3}$ concerns the comparability of heart rates analysed in the SIDS cases and their controls. Figure 5 of their study showed that the five recordings from SIDS cases that showed the lowest values for $b$ also had higher average heart rates than controls. In the present study we compensated for the significantly higher heart rates previously reported to be present in these particular recordings from SIDS cases ${ }^{78}$ by selecting segments in both controls and SIDS cases where the range of heart rates was as wide as possible (for example, by including data from the whole 24 hour period) and by matching our controls for birth weight and postnatal age.

Sadeh et al reported a linear relation "significantly different from zero at $P=0.0002$ " for their plot of mean RR intervals against mean values of $b$ in their control group. ${ }^{3}$ In our analysis no such linear relation was seen between mean $b$ and mean $R R$ for our controls (see 
fig 2A), thus supporting our use of a power equation. It is difficult to understand how Sadeh $e t a l^{3}$ can reconcile their findings of a linear relation and continue to use the original power law equation where $b$ is assumed to be constant with respect to RR.

In conclusion, as in our earlier reports, ${ }^{12}$ we found no evidence to support the hypothesis that a defect in ventricular repolarisation time or its relation to heart rate are predictive for sudden and unexplained infant deaths. We can rule out neither the unlikely possibility that a serious repolarisation abnormality was present that could not be detected by surface electrocardiogram nor the possibility that a subsequent defect in repolarisation developed closer to the time of death.

We thank Mrs Joyce Lamb for her help in preparing these data for analysis, Dr A J Wilson for his early suggestions on apparen heart rate effects on QT intervals resulting from the inherent heart rate effects on QT intervals resulting from the inherent phase response of high pass filters, and to $\mathrm{Dr} W \mathrm{New}$ and
Professor R H Anderson for their expert advice on this paper.
This project and DPS were funded by Nellcor and the National Heart and Chest Hospitals.

1 Southall DP, Richards JM, de Swiet M, Arrowsmith WA Cree JE, Fleming PJ, et al. Identification of infants destined to die unexpectedly during infancy: evaluation of predictive importance of prolonged apnoea and disorders of cardiac rhythm or conduction. First report of a multicentred prospective study into the sudden infant death syndrome. BMJ 1983;286:1092-6.

Southall DP, Arrowsmith WA, Stebbens V, Alexander JR. QT interval measurements before sudden infant death QT interval measurements before sudden

3 Sadeh D, Shannon DC, Abboud S, Saul JP, Akselrod S Cohen RJ. Altered cardiac repolarization in some victims of sudden infant death syndrome. $N$ Engl J Med 1987; of sudden

4 Wynn VT. Heart rate dependence of the RT interval in the first month of life. Clin Phys Physiol Meas 1986;7:369-73. 5 Wynn VT. Measurement and analysis of RT and RR intervals in 72 infants in the age range 2 to 75 days. In Copeland $\mathrm{K}$, ed. Progress reports on electronics in medicine and biology. The Institution of Electronic and Radio Engineers, 1986:331-6. ISBN 0903748657.

6 Bundy K, Wynn VT. ECG tape-speed error compensator. Med Biol Eng Comput 1985;23:607-9.

7 Wilson AJ, Stevens V, Franks CI, Alexander JR, Southall DP. Respiratory and heart rate patterns in infants destined to die of the sudden infant death syndrome. BMJ to die of the sudd

8 Southall DP, Stevens V, Franks CI, Newcombe RG, Wilson AJ. Sinus tachycardia in term infants preceding sudden Aj. Sinus tachycardia in term infants preceding sudd

9 Bazett $H C$ An analysis of the time-relations of electrocardiograms. Heart 1920;7:353-70. 